\title{
Cicatrización de úlceras venosas con difenilhidantoinato de sodio.
}

\section{Artículo Original}

Martha A.Castañeda-Vázquez ${ }^{1}$, Hugo Castañeda-Vázquez² ${ }^{2}$ Pedro Garzón de la Mora ${ }^{1}$, Natividad FloresMartínez ${ }^{3}$.

${ }^{1}$ Centro de Investigación Biomédica de Occidente, Instituto Mexicano del Seguro Social, Guadalajara, Jalisco. ${ }^{2}$ Departamento de Salud Publica, Centro Universitario de Ciencias Biológicas y Agropecuarias, Universidad de Guadalajara, Zapopan, Jalisco. ${ }^{3}$ Centro Medico de Occidente, Instituto Mexicano del Seguro Social; Guadalajara, Jalisco, México.

\section{RESUMEN.}

Introducción. Las úlceras venosas de extremidades inferiores muestran una incidencia de 2.5 millones de casos anuales en los Estados Unidos y una prevalencia de 22 por cada 100 personas. Además son recurrentes, incapacitantes y repercuten en forma severa para la deambulacion. Un medicamento utilizado en casos de epilepsia, el Difenilhidantoinato de sodio (DFH-Na), puede favorecer la cicatrización de las úlceras venosas.

Material y métodos. Seleccionamos 20 pacientes que presentaron ulceración de la pierna con diagnostico de secuela postflebitica, a 10 de esos pacientes se les aplico diariamente DFH-Na, por vía tópica y por espacio de 2 a 3 meses en las úlceras, previo aseo con agua y jabón. Los diez pacientes restantes correspondieron al grupo testigo que recibió tratamiento convencional (agua y jabón. Se evaluaron parámetros generales en los 20 pacientes (sexo, edad, ocupación, antecedentes sobre anticonceptivos, patologías asociadas, tiempo de aparición de la úlcera, recidivas), así como tomas de biopsias, fotografías y mediciones planimétricas al inicio y final del tratamiento.

Resultados. Al final del tratamiento observamos que el $40 \%$ de los pacientes respondió al DFH-Na como inductor de la cicatrización y ninguno al tratamiento ordinario. En el primer grupo se desarrolló tejido de granulación y desplazamiento epitelial hacia las soluciones de continuidad de la piel.

Conclusiones. La aplicación tópica del DFH$\mathrm{Na}$, como recurso farmacológico para la cicatrización de úlceras venosas resulta favorecedor por disminuir el tiempo de curación

Solicitud de sobretiros: Dra. Martha Castañeda-Vázquez, Av. Plan de Ayala 1856-5, Col. Parques del Auditorio, C.P. 45190 Zapopan, Jalisco, México. E-mail: hcastane@maiz.cucba.udg.mx. 
MACastañeda-Vázquez, H Castañeda-Vázquez, P Garzón de la Mora, N Flores-Martínez.

y disminución en el costo del tratamiento.

(Rev Biomed 2001; 12:148-156)

Palabras clave: Úlceras venosas, difenilhidantoinato de sodio, cicatrización, extremidades inferiores.

\section{SUMMARY.}

Cicatrization of venous ulcers with dyphenilhydantoin sodium.

Introduction. Venous ulcers of lower extremities show an incidence of 2.5 million cases annually in the United States of America and a prevalence of 22 per 100 people. They are recurrent, cause incapacity and can also cause ambulatory problems. Topical application of dyphenylhydantoin sodium can favor the scarring of the above mentioned ulcers.

Material and Methods. 20 patients with leg ulceration were selected. The diagnosis was posflebitic sequel. We applied topical DFH-Na to the ulcers on a daily basis for 2 to 3 months, after washing with soap and water. The ten remaining patients received conventional treatment (washing with soap and water). General data of the 20 patients: was evaluated sex, age, employment, record of contraception, associated pathologies, ulcer apparition time, relapses. We took biopsies, photographs and carried out planimetric halftion at the beginning and at the end of treatment, elaborating comparative data.

Results. We observed that $40 \%$ of the patients responded to DFH treatment and in none of the control patients was improvement seen in the healing of flebostatic ulcer. Granulation tissue and epithelial displacement was developed in the DFH-Na group. In the group which received conventional treatment no improvement was observed and 20\% developed a light infection. Conclusions. Our results demonstrate the effectiveness of DFH-Na to induce cicatrisation of venous ulcers.
(Rev Biomed 2001; 12:148-156)

Key words: Venous ulcers, dyphenilhydantoin sodium, cicatrization, lower extremities.

\section{INTRODUCCIÓN.}

El incremento en la presión hidrostática y el éxtasis venoso prolongado origina úlceras en piel, predominantemente de extremidades inferiores, cuya extensión depende de la magnitud de la oclusión o dificultad del retorno sanguíneo $(1,2)$.

Las causas desencadenantes de úlceras venosas son la oclusión trombotica o residual, así como la presencia de válvulas venosas incompetentes. La trombosis venosa profunda es más frecuente y produce con mayor incidencia de ulceración de las piernas (2-4). La recuperación se logra a través de medidas que eviten la hipertensión venosa, infecciones y medicamentos tales como flúor hidrocortisona, neomicina o cloranfenicol (3).

Hasta el momento no existe medicamento alguno que sea eficaz para resolver las consecuencias de las oclusiones venosas que originan solución de continuidad en la piel. Un recurso farmacológico para la solución de estas úlceras podría ser la aplicación de difenilhidantoinato de sodio (DFH-Na) en virtud del incremento de tejido de granulación por estimulo directo en la fibroblastogenésis (5).

Antes que el DFH fuera aprobado para el tratamiento de cualquier otro tipo de trastorno o padecimiento, fue clasificado como anticonvulsionante. Su síntesis química se logró en 1908 y su actividad anticonvulsivonante fue demostrada hasta 1938 por Merritt y Putnam (6). Sus propiedades principales y más estudiadas corresponden a la capacidad del fármaco para limitar el desarrollo de la actividad máxima de las crisis y para reducir la disfunción de las crisis desde un foco

\section{Revista Biomédica}


activo.

Un estudio estomatológico realizado en 58 pacientes, indica que los efectos histológicos y clínicos del DFH-Na con masaje diario gingival en periodontopatías, induce cambios histológicos y clínicos que confirman la acción fibroblastogénica del DFH-Na y la cicatrización con esclerosis. Además presenta disminución en la infiltración inflamatoria (7). En otro estudio con 80 ratas se encontró que el DFH-Na acelera la cicatrización de fracturas inestables mediante la formación del callo óseo (8).

Por lo tanto, nuestro trabajo pretende esclarecer sí la fibroblastogenesís inducida por DFH-Na permite la cicatrización de úlceras de extremidades inferiores ocasionadas por isquemia tisular.

\section{MATERIAL Y MÉTODOS. Recursos Humanos.}

Humanos: Médico especialista en Angiologia y cirugía cardiovascular; Médico especialista en Anatomía patológica, Médico especialista en Medicina Interna y Bioquímica, Médico pasante y auxiliar de enfermera general.

\section{Materiales:}

Formato de recolección de datos.

Instalaciones del Hospital (consultorio y sala de curaciones).

Laboratorio de Histopatología.

Cámara Fotográfica y Planímetro.

Difenilhidantoinato de sodio.

Agua y Jabón.

\section{Métodos.}

Esta investigación se llevó en la consulta externa del servicio de Angiología del Hospital de Especialidades del Centro Medico de Occidente del IMSS. Se incluyeron 20 pacientes que acudieron a la consulta externa de angiologia, con diagnóstico de secuela postflebítica y que presentaron ulceración en el tercio distal de la pierna y sin evidencia aparente de infección (material purulento).

Con los 20 pacientes se formaron 2 grupos, los 10 primeros integraron el grupo I y con los 10 restantes se formó el grupo II.

El grupo I (control), incluyó 10 pacientes a los cuales diariamente se les realizaron aplicaciones tópicas de $200 \mathrm{mg}$ de difenilhidantoinato de sodio, por espacio de 8 a 12 semanas, previo lavado con agua, jabón y secado con gasa estéril, lo cual se llevó a cabo en la sala de curaciones anexa al consultorio de Angiología. La dosificación inicial del DFH$\mathrm{Na}$ originó quemaduras en el tejido expuesto (centro de la úlcera) en algunos casos, por lo cual fue necesario modificar la dosis del compuesto; sin embargo se aplicó la cantidad necesaria para formar una capa de DFH-Na que cubriera el área de la lesión, por lo que fue disminuyendo la cantidad aplicada según disminuía el área de la úlcera.

El grupo II incluyó a 10 pacientes a los cuales se les realizo únicamente lavado con agua y jabón y secado con gasa estéril diariamente por un periodo de 8 a 12 semanas.

A $\operatorname{los} 20$ pacientes en general se les practicó una encuesta con parámetros generales que incluyeron datos sobre género, edad, ocupación, tabaquismo antecedentes quirúrgicos y gineco-obstétricos, tiempo de aparición de la úlcera, recidiva, entre otras.

Se obtuvieron biopsias antes de iniciar el tratamiento y al finalizar el mismo, para determinar la reparación de la ulceración. Para evaluar la evolución microscópica de la cicatrización en los 20 pacientes, se les practicaron mediciones de superficie, así como tomas fotográficas al inicio y al finalizar el tratamiento.

Se determinaron los niveles sanguíneos de DFH-Na a las 4 semanas de tratamiento a pacientes del grupo I. Se excluyeron aquellos 
MACastañeda-Vázquez, H Castañeda-Vázquez, P Garzón de la Mora, N Flores-Martínez.

pacientes no cooperadores o que presentaban signos de infección en la úlcera a la inspección visual. Una vez que se tuvieron recolectados los datos se realizó la clasificación en forma cuantitativa y cualitativa.

Las superficies recuperadas en las úlceras, resultado de la aplicación de DFH o agua y jabón, se compararon a través de la prueba estadística ANOVA.

\section{RESULTADOS.}

Los datos obtenidos de los 20 pacientes estudiados mostraron como causa desencadenante de úlcera venosa a la trombosis venosa profunda en el $70 \%$ de los casos, predominando éstas en el sexo femenino (65\%), con un intervalo de edad de 31 a 79 años.

Se trataron ulceraciones que presentaron tiempo de aparición de 30 días a 34 años, 4 de evolución corta (de 30 días a 12 meses) y 16 de evolución larga (de 13 meses a 34 años), de las cuales el $85 \%$ de ellas presentaron recidivas durante el tiempo de evolución a pesar de ser tratadas con múltiples esquemas terapéuticos (antibióticos, antiinflamatorios, extractos de plantas medicinales, cicatrizantes) en el $95 \%$ de los casos (cuadro 1).

Las fotografías tomadas al inicio, transcurso y finalización del tratamiento en ambos grupos permitieron valorar la evolución macroscópica de la cicatrización (cuadro 2), en donde se observaron granulaciones difusas en el tejido central de la ulceración desde el segundo día de iniciado el tratamiento con DFH-Na (figura 1) en comparación con el grupo tratado con agua y jabón.

Asimismo, observamos un incremento en el transudado de la ulceración en las primeras dos semanas del tratamiento con DFH-Na. Hubo aumento en la vascularización, determinada por hiperemia y pequeñas hemorragias observadas al retirar el apósito en la 5-6a. semana de tratamiento con DFH$\mathrm{Na}$ (figura 2), no así en los pacientes que fueron tratados con agua y jabón, los cuales presentaron en algunos casos aparición de granulaciones en la región central de la úlcera entre la 5a-6a. semana de tratamiento (figura $3)$.

Al finalizar el tratamiento se obtuvo cicatrización total en el $40 \%$ de los pacientes tratados con DFH-Na, que presentaron úlceras mayores de 94.80 a $10.44 \mathrm{~cm}$ (figuras 4), en las úlceras mayores de 52.14 a $477.70 \mathrm{~cm}$ se obtuvieron mediciones de hasta $4 \mathrm{~cm}$ de tejido fibroso en el borde de la úlcera.

Ninguno de los pacientes del Grupo II (tratados con agua y jabón) mostró cicatrización total al finalizar el tratamiento. Encontramos reducción total del tamaño de la úlcera en el $20 \%$ de los pacientes con úlceras menores de $89.64 \mathrm{~cm}$, así como incremento del mismo en el $20 \%$ de los casos con úlceras mayores de $52.14 \mathrm{~cm}$.

Hubo aparición de signos clínicos de infección en el 20\% de los pacientes del grupo tratado con agua y jabón, en cambio ninguno en los pacientes del Grupo I.

Se tomaron biopsias al principio y al final del tratamiento en los pacientes de ambos grupos (cuadro 3). Las biopsias obtenidas antes de iniciar el tratamiento revelaron: piel ulcerada con borde necrotico; infiltrado inflamatorio agudo; escaso tejido fibroconenctivo dérmico.

Al finalizar el tratamiento en las biopsias obtenidas en los pacientes tratados con agua y jabón se encontró: necrosis superficial; fragmentos de tejido fibroso; proliferación vascular; infiltrado inflamatorio agudo.

En los pacientes tratados con DFH-Na en las biopsias obtenidas al finalizar el tratamiento observamos: borde de úlcera de piel con granuloma reparativo: hiperplasia pseudoepiteliomatosa de la epidermis; fibrosis dérmica; inflamación crónica multifocal;

\section{Revista Biomédica}


Tratamiento de úlceras venosas.

Cuadro 1

Parámetros Generales

Frecuencia de trombosis venosa profunda y datos demográficos en 20 pacientes con úlcera flebotómica.

\begin{tabular}{lccr}
\hline & $\begin{array}{c}\text { DFH-Na } \\
(\mathrm{n}=10)\end{array}$ & $\begin{array}{c}\text { Agua y Jabón } \\
(\mathrm{n}=10)\end{array}$ & Total (\%) \\
\hline $\begin{array}{l}\text { Trombosis } \\
\text { Venosa } \\
\text { Profunda }\end{array}$ & 7 & 10 & $17(85)$ \\
Sexo & & & \\
$\begin{array}{l}\text { Femenino } \\
\text { Masculino }\end{array}$ & 6 & 7 & $13(65)$ \\
$\begin{array}{l}\text { Intervalo } \\
\text { de Edad }\end{array}$ & $37-39$ años & $31-73$ años & \\
$\begin{array}{l}\text { Tiempo de } \\
\text { Aparición }\end{array}$ & & 35 días a 34 años & 0 a 11 años \\
\hline
\end{tabular}

DFH-Na = Difenilhidantoina sódica.

proliferación vascular telangiectásica; proliferación de tejido conectivo.

Las mediciones planimétricas que se efectuaron al inicio del tratamiento en ambos

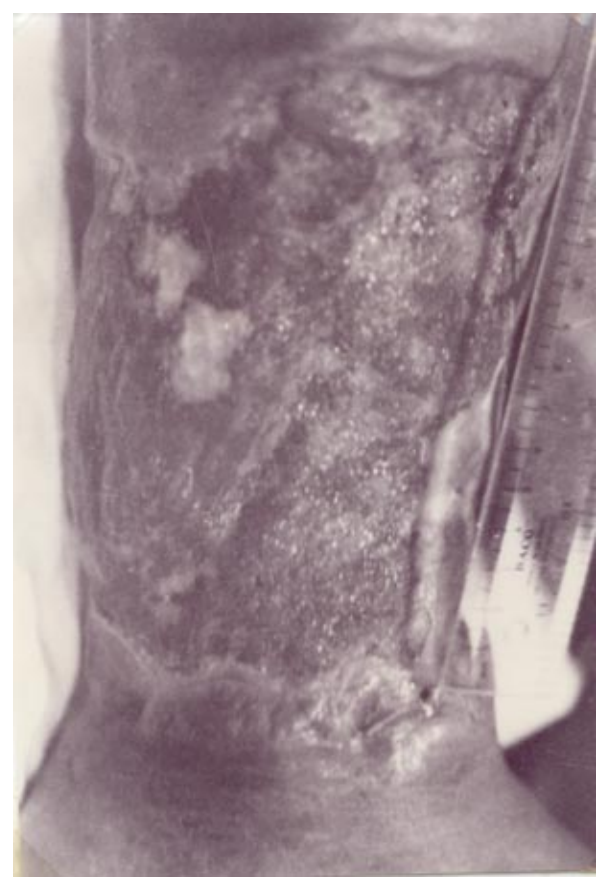

Figura 1.- Tejido de Granulación obtenido al segundo día de tratamiento con DFH-Na.

\section{Cuadro 2}

Evaluación Macroscópica de la evolución de úlcera venosa tratadas con DFH-Na, agua y jabón.

\begin{tabular}{lcc}
\hline & DFH-Na & Agua y Jabón \\
\hline Total de casos & $(\mathrm{n}=10)$ & $(\mathrm{n}=10)$ \\
Cicatrización total & 4 & 0 \\
Signos de Infección & 0 & 2 \\
$\begin{array}{l}\text { Incremento de tejido } \\
\text { de Granulación }\end{array}$ & +++ & + \\
Aumento del transudado & ++ & + \\
\hline
\end{tabular}

+ bajo; ++ medio; +++ elevado.

$\mathrm{DFH}-\mathrm{Na}=$ Difenilhidantoina sódica.

grupos para conocer el área real de la superficie tisular, en comparación al área real de superficie al finalizar el tratamiento, mostraron que en pacientes del grupo I (tratados con DFH-NA) el total del porcentaje

Cuadro 3

Efecto del DFH-Na y del agua y jabón en la evolución microscópica de úlceras venosas.

\begin{tabular}{lcc}
\hline & DFH-Na & Agua y Jabón \\
\hline Pre-tratamiento & & \\
Infiltrado Inflamatorio & Agudo & Agudo \\
Reborde Necrotico & ++ & ++ \\
Tejido Fibroconectivo & Escaso & Escaso \\
Post-tratamiento & & \\
Infiltrado Inflamatorio & Crónico & Agudo \\
Proliferación Vascular & +++ & + \\
Tejido Fibroconectivo & +++ & + \\
\hline
\end{tabular}

DFH-Na $=$ Difenilhidantoina sódica.

$+=$ bajo

$++=$ intenso

Vol. 12/No. 3/Julio-Septiembre, 2001 
MACastañeda-Vázquez, H Castañeda-Vázquez, P Garzón de la Mora, N Flores-Martínez.

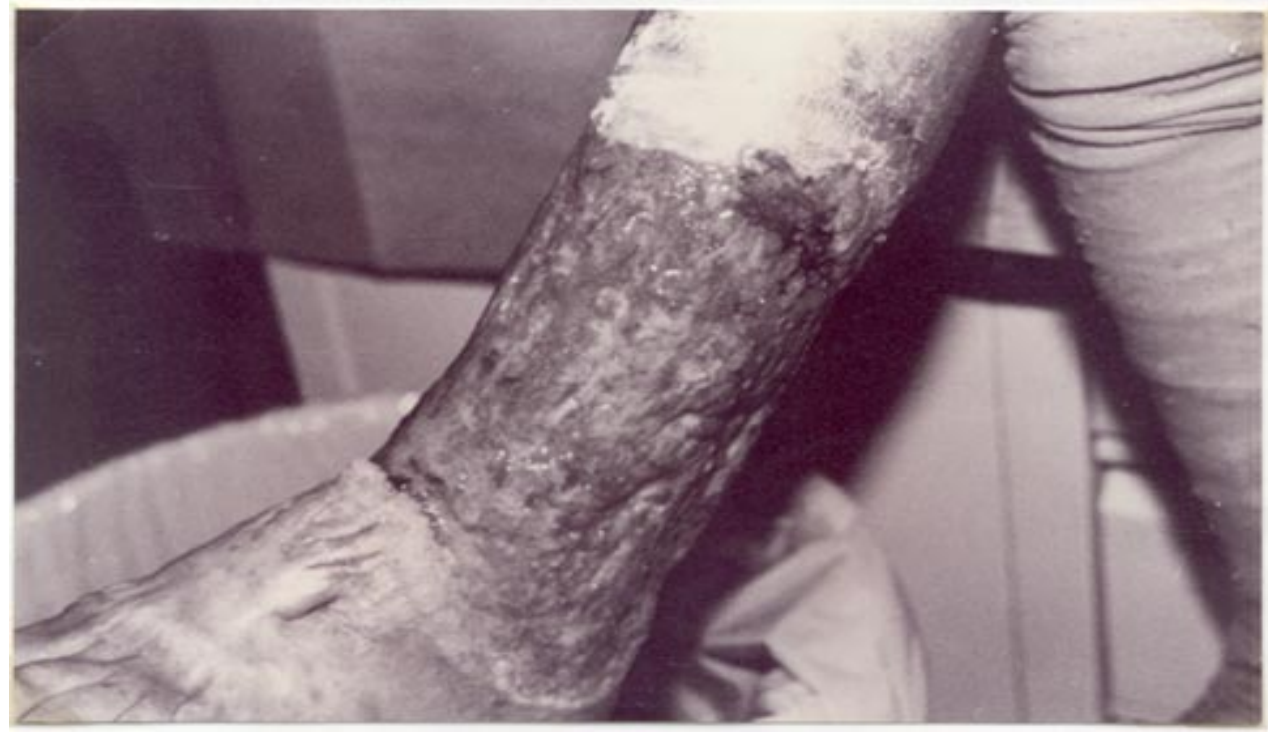

Figura 2.- Aumento en la vascularización 5-6a. Semana de tratamiento con DFH-Na. Final del tratamiento: note el tejido cicatrizal en el borde superior de la úlcera.

recuperado fue del $38.24 \%$ a diferencia del total de porcentaje recuperado en pacientes del grupo II que resultó ser del $17.10 \%(\mathrm{p}<0.005)$ (cuadros 4 y 5 ).

Los niveles sericos del DFH-Na correspondieron a un rango de 1 a $2 \mathrm{~g} / \mathrm{mL}$ y promedio de $1.5 \mathrm{~g} / \mathrm{mL}$. Habitualmente corresponde a $10-20 \mathrm{~g} / \mathrm{mL}$ el rango terapéutico en pacientes que ingieren DFH-Na.

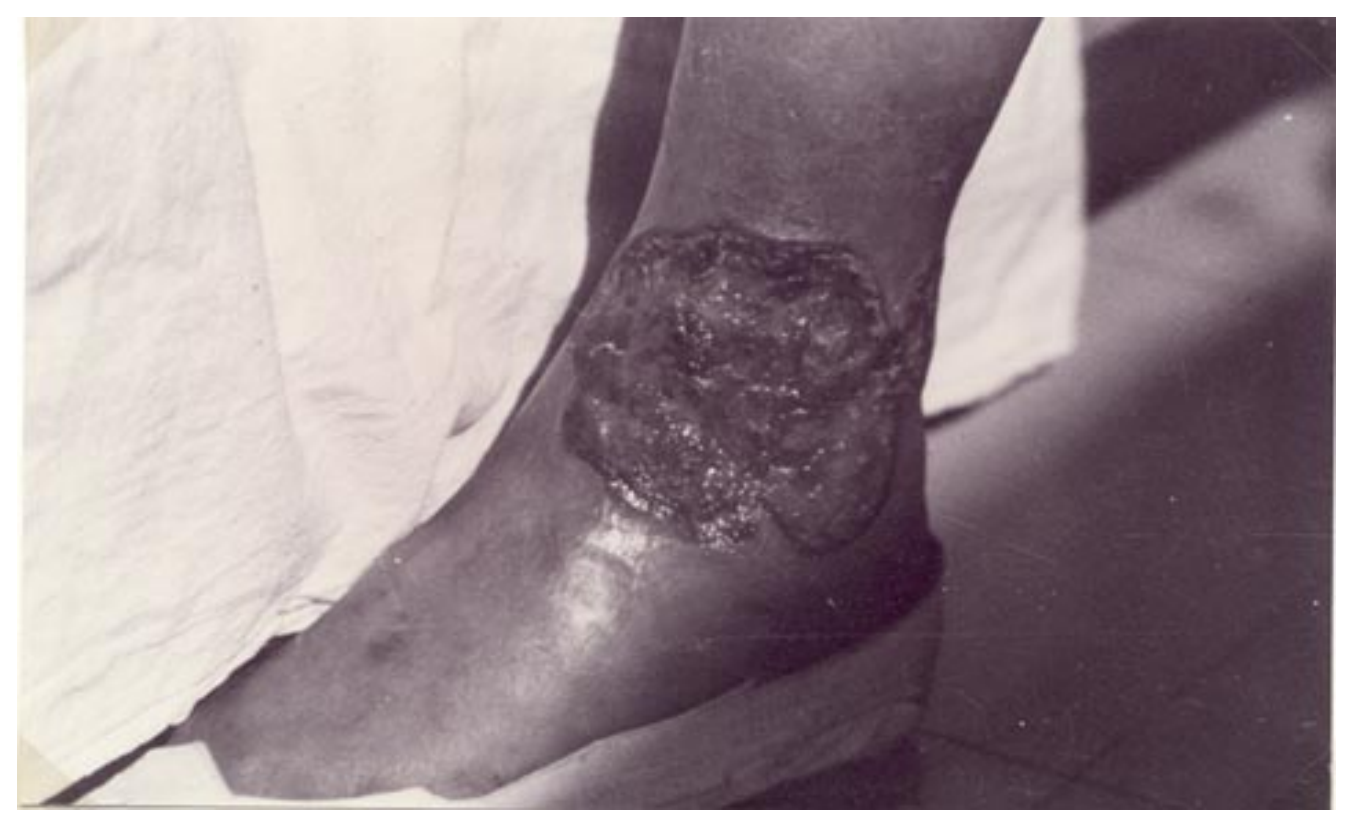

Figura 3.- Incremento del área ulcerada, secundario a infección agregada en paciente tratado con agua y jabón. 
Tratamiento de úlceras venosas.

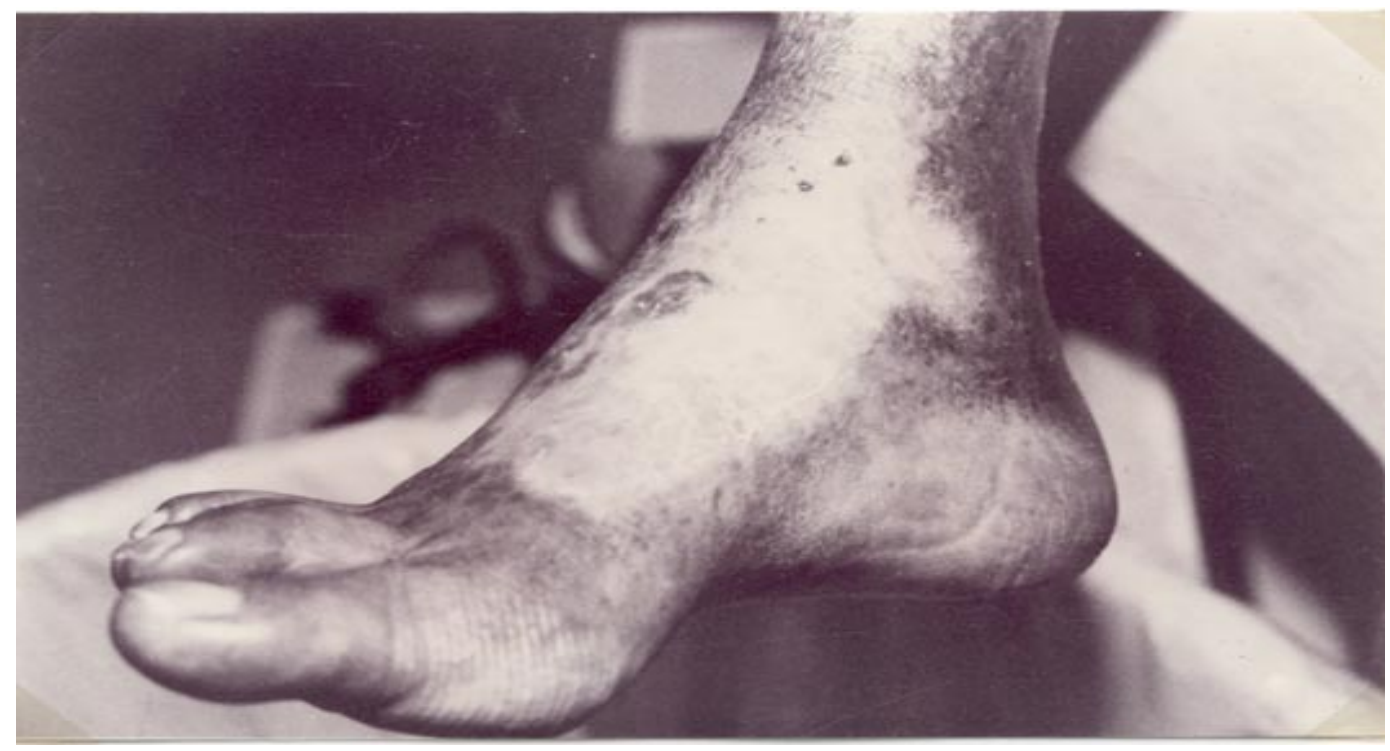

Figura 4.- Cicatrización total de úlcera tratada con DFH-Na, al finalizar el tratamiento.

\section{DISCUSIÓN.}

El Difenilhidantoinato de sodio es un medicamento antiepiléptico, eficaz para el control de las crisis convulsivas. Desde su descubrimiento en 1937, ha demostrado su capacidad de inducir múltiples efectos colaterales (6). Algunos son indeseables como la hiperplasia gingival, según puede inferirse de diversos experimentos en animales, y en el humano. De esta manera, el fármaco induce una reducción significativa en la actividad de la colagenaza y fibrasa de la colágena (9-12). En piel de ratas la aplicación de DFH-Na no afecta la cantidad de colágena, sin embargo aumenta el tejido de granulación en $21 \%$ (10). En ratones fracturados experimentalmente, este

Cuadro 4

Superficie recuperada $\left(\mathrm{en}^{2} \mathrm{~cm}^{2}\right)$ de úlceras venosas de 10 pacientes tratados con agua y jabón.

\begin{tabular}{cccccc}
\hline Paciente & Inicio & Final & Diferencia & \% de Recuperación* & Recuperación diaria* \\
\hline 1 & 5.98 & 5.98 & 0 & 0 & 0 \\
2 & 14.64 & 12.46 & 2.18 & 14.9 & 0.2 \\
3 & 22.46 & 17.47 & 4.99 & 22.22 & 0.5 \\
4 & 54.14 & 47.38 & 4.76 & 9.2 & 0.56 \\
5 & 58.36 & 48.30 & 10.6 & 17.24 & 0.12 \\
6 & 60.58 & 51.80 & 8.78 & 14.5 & 0.10 \\
7 & 89.64 & 89.22 & -67.42 & 0.47 & 0 \\
8 & 105.16 & 178.28 & 10.11 & -63.82 & 0.12 \\
9 & 283.51 & 273.40 & 0 & 28.72 & 0 \\
10 & 292.38 & 292.38 & 0 & \\
\hline
\end{tabular}

* El promedio del \% de recuperación de superficie fue de $4.34 \%$ y el de la recuperación diaria de $0.16 \mathrm{~cm}^{2}$. 
MACastañeda-Vázquez, H Castañeda-Vázquez, P Garzón de la Mora, N Flores-Martínez.

Cuadro 5

Superficie recuperada $\left(\mathrm{en}^{\left.\mathrm{cm}^{2}\right)}\right.$ de úlceras venosas de 10 pacientes tratados con DFH-Na

\begin{tabular}{cccccc}
\hline Paciente & Inicio & Final & Diferencia & \% de Recuperación* & Recuperación diaria* \\
\hline 1 & 10.44 & 7.38 & 3.06 & 29.38 & 0.3 \\
2 & 11.54 & 4.54 & 7.0 & 60.66 & 0.8 \\
3 & 19.76 & 10.56 & 9.2 & 46.56 & 0.10 \\
4 & 21.40 & 10.64 & 10.76 & 50.29 & 0.12 \\
5 & 34.26 & 13.58 & 20.68 & 60.37 & 0.24 \\
6 & 94.80 & 11.48 & 83.32 & 87.9 & 0.99 \\
7 & 292.36 & 236.76 & 28.6 & 9.79 & 0.34 \\
8 & 347.22 & 277.72 & 68.6 & 20.04 & 0.81 \\
9 & 373.82 & 338.34 & 35.48 & 9.5 & 0.42 \\
10 & 477.70 & 439.62 & 38.08 & 7.98 & 0.45 \\
\hline
\end{tabular}

* El promedio del \% de la recuperación de superficie fue de $38.24 \%$ y el de la recuperación diaria de $0.45 \mathrm{~cm}^{2}$.

medicamento induce una rápida regeneración de tejido óseo a través de un incremento en la síntesis de colágeno (8).

Los resultados obtenidos en el presente estudio de cicatrización de úlceras venosas con DFH-Na, en donde se manifiesta el incremento en la proliferación de tejido fibroconectivo y disminución en el tiempo de cicatrización, podrían explicarse por el efecto inductor de la fibroblastogénesis y así favorecer los procesos de cicatrización.

Las úlceras venosas son complicaciones frecuentes que producen incapacidad funcional, por su tendencia a la cronicidad, infecciones secundarias, recidivas constantes y curación rebelde, repercuten en los aspectos socioeconómicos de las personas que lo padecen así como de la familia. La aplicación tópica del DFH-Na, como recurso farmacológico para la cicatrización de úlceras venosas por sus efectos sobre tejido colágeno, resulta favorecedor por disminuir el tiempo de curación y como consecuencia disminución en el costo del tratamiento.

\section{REFERENCIAS.}

1.- Olivencia JA. Pathophysiology of venous ulcers: surgical implications, review and update. Dermatol Surg 1999;25:880-5.

2.- Smith PD. Update on chronic-venous-insufficiencyinduced inflamatory processes. Angiology 2001; 52 (suppl 1): S35-S42.

3.- Clement DL. Venous ulcer reappraisal: insights from an international task force. J Vasc Res 1999; 36 (suppl 1): 42-7.

4.- Angle N, Bergan JJ. Chronic venous ulcer. Br Med J 1997; 314:1019-23.

5.- Dormandy JA. Pharmacologic treatment of venus leg ulcers. J Cardiovasc Pharmacol 1995; 25 (suppl 2):S61-S5.

6.- McNamara JO. Fármacos eficaces para el tratamiento de las epilepsias. En Hardman JG, Limbird LE, Molinoff PB, Ruddon RW, Goodman-Gilman A, editores. Las bases farmacológicas de la terapéutica. $9^{a}$ Ed. México: McGraw-Hill Interamericana; 1997. p. 491-519.

7.- Chicani P. Use of sodium phenytoin in the treatmet of periodontal diseases; Actal Odontostomatol 1972; 26: $265-74$.

8.- Gudmunson C, Lidgren L. Does diphenylhydantoin acelerate healing of fracture in mice. Acta Orthop Scand 1973; 44:640-9.

\section{Revista Biomédica}


9.- Ariyoshi T, Remmer H. Die wirkung von verschiedene fettfraktionen der leber. Naunyn schieedeberg. Arch Pharm Exp Path 1968; 260:90-1.

10.- Bazin S, Delaunay. Action exercee par des substances lathyrogenes sur le collagene des granulomes. CR Acad Sci (Paris) 1972; 274:18-20.

11.- Donoff B. The effect of diphenylhydantoin on open wound healing on guinea pigs. J Surg Res 1978; 24: 45-4.

12.- Goultschin J, Soshan S. Inhibition of collagen breakdown by diphenylhydantoin. J Biochem Biophys Acta 1980; 631:189-91. 